\title{
Recording eye movements with video-oculography and scleral search coils: a direct comparison of two methods
}

\author{
J.N. van der Geest *, M.A. Frens \\ Department of Physiology, Erasmus University Rotterdam, P.O. Box 1738, 3000 DR, Rotterdam, The Netherlands
}

Received 24 September 2001; received in revised form 3 December 2001; accepted 7 December 2001

\begin{abstract}
A video-based 2D eye-tracking system (EyeLink version 2.04, SR Research Ltd/SMI) was compared with the scleral search coil technique for its performance on recording the properties of fixations and saccadic eye movements. Fixation positions and saccadic properties (amplitude, duration, and peak velocity) were calculated independently from the data of the two systems that recorded eye positions simultaneously. Fixation positions were well correlated between the video and the coil output with an average discrepancy of $<1^{\circ}$ over a tested range of 40 by $40^{\circ}$ of visual angle. With respect to the saccade analysis, the values measured by the video system were fitted as a linear function of the values measured by the coil system. Highly correlated linear fits with slopes near one were obtained for all the saccadic parameters. Main sequence relationships (amplitudes-duration and amplitude-peak velocity) were also similar for both systems. A disadvantage of the video method is its low sample rate of 250 $\mathrm{Hz}$. The relatively noisier estimate of all parameters of small saccades could be attributed to this low sampling frequency. (C) 2002 Elsevier Science B.V. All rights reserved.
\end{abstract}

Keywords: Eye movement; Recording technique; Search coil; Video-oculography; Saccade; Fixation; Human

\section{Introduction}

The scleral search coil method (Robinson, 1963; Collewijn et al., 1975; Remmel, 1984) is widely used to measure the position of the eye. Eye position is determined by placing a silicon annulus in the eye. This annulus contains a coil of thin copper wire. When the subject is placed in an a.c. magnetic field, the position of the eye can be determined from the amplitude of the induction current in the coil. The low noise of the coil method allows for high spatial $\left(\ll 1^{\circ}\right)$ and temporal $(\ll 1 \mathrm{~ms}$ ) resolutions and therefore, the coil method is regarded as the gold standard in oculomotor research (Collewijn, 1998).

However, one of the major disadvantages is the invasive nature of this method. The experimental time is limited to about $30 \mathrm{~min}$, since most subjects cannot bear a coil in their eye for a longer period, even when

\footnotetext{
* Corresponding author. Tel.: + 31-10-408-7556; fax: + 31-10-4089457.

E-mail address: vandergeest@fys.fgg.eur.nl (J.N. van der Geest).
}

the eye is anesthetized. Therefore, it is difficult to get full cooperation of the subjects, especially children, patients, and weak-hearted colleagues.

Recent technological advances have produced noninvasive two-dimensional eye-tracking devices that are capable of recording the position of the eye with high spatial and temporal resolutions. These resolutions allow, in theory, to determine the metric and kinematical properties of saccadic eye movements with the same precision as with the coil method.

In this paper, the properties of saccadic eye movements as measured by a video-based 2D eye-tracking device (the Eyelink system, developed by SR Research Ltd, currently marketed by SensoMotoric Instruments (SMI) GmbH, Germany) are compared to the properties as measured with the scleral search coil. Furthermore, fixation positions are compared between the two systems. These two eye movements allow for a comparison of the tracking capabilities of the video system with the coil method under both static and highly dynamic conditions. Other types of eye movements, such as smooth pursuit and optokinetic and vestibular 
reflexes, were not evoked since we used a static environment in combination with the use of a bite-board. However, by exploring fixations and saccades the whole frequency spectrum of all possible movements from DC to $100 \mathrm{~Hz}$ are covered.

Prior comparisons between the scleral coil method and non-invasive recording methods have so far concluded in favor of the coils, either because of inherent non-linearities during saccades (SRI Purkinje Tracker; Deubel and Bridgeman, 1995), or because sample rates are too low (EL-MAR video system; DiScenna et al., 1995). On the other hand, it has been argued that the coil-induced discomfort can affect the normal movements of the eye, as in mice (Stahl et al., 2000).

\section{Methods}

\subsection{Subjects}

Four healthy male volunteers participated in the experiment. Left and right eye recordings were made from subjects A, B and C; left eye recordings only were made from subject $\mathrm{D}$, who is monocular and has a prosthesis in his right eye. Subject C wore contact lenses and subject $\mathrm{D}$ wore glasses during the experiment. All subjects were highly familiar with the wearing of scleral search coils. Subjects A and B are the authors.

The irises of subjects $\mathrm{A}, \mathrm{B}$ and $\mathrm{C}$ are blue, and subject $\mathrm{D}$ has a brown iris. The color of the iris had no influence on the performance of the video system.

\subsection{Apparatus}

A NEC-Multisync X21 21" computer monitor was used for presenting calibration targets and stimuli. The monitor was positioned $50 \mathrm{~cm}$ in front of the subject and the center of the screen was horizontally and vertically aligned with the center between the two eyes.

Two-dimensional (2D) eye movements were recorded simultaneously, but otherwise completely independently, with a video-based system (EyeLink system version 2.04, SR Research Ltd/SMI GmbH, Germany) and a scleral search coil system (Remmel Labs, USA, with coils from Skalar, Delft, NL). Sample rate was 250 $\mathrm{Hz}$ for the video system and set at $500 \mathrm{~Hz}$ for the coil system. The range of the coil system was set to $\pm 20^{\circ}$ horizontally and vertically. The coil and video signals were recorded on separate computers and aligned in time later.

The EyeLink system is a video-based, head-mounted eye-tracking device that uses two miniature infrared cameras to track both eyes simultaneously. Eye recordings are controlled using specific hardware and software provided by the manufacturer (version 2.04). The dark pupil system tracks the center of the pupil by an algorithm similar to a centroid calculation with a theoretical noise-limited resolution of $0.01^{\circ}$ and velocity noise of $<3^{\circ}$ s for two-dimensional eye tracking (details provided by SR Research Ltd). The implemented heuristic filtering (Stampe, 1993), which according to the developer removes single-sample artifacts and does not affect measured saccade characteristics, was disabled, yielding an average delay from eye movements to position data availability of about $6 \mathrm{~ms}$. Please note that in contrast to many other video systems, this video system does not track a corneal reflection.

The EyeLink system is also equipped with a head movement tracker. This was not tested in the present study, since the head was immobilized with the use of a bite-board.

\subsection{Calibration}

The video system was calibrated using the built-in nine dot routine. In short, the subject had to fixate nine subsequent dots that appeared in random order at 3 by 3 evenly spaced locations on the monitor. The manufacturer's software, that maps pupil position to a headreferenced coordinate frame using a biqaudratic mapping function (personal communication with SR Research Ltd.; Sheena and Borah, 1981; Stampe, 1993), produced the subsequent output of the eye position both horizontally and vertically in pixels of the stimulus monitor. These values were converted to Euler angles, taking the distance between the eye and the monitor into account.

Search coils were calibrated before inserting them into the eye. The coils were mounted on a gimbal system and were placed in the center of the magnetic fields. Sensitivity of the coils was determined by rotating them over $10^{\circ}$ horizontally and vertically. After placing the coils in the eye, the subject was asked to fixate the center of the monitor and offsets were removed from the coil signal.

\subsection{Stimuli}

Three different experimental paradigms were tested, labeled 'Target', 'Light', and 'Dark'.

In the 'Target' paradigm, two black dots were displayed on a uniform light-gray background. The position of the dots was centered around the central point of the screen. The visual angle between the dots (target amplitude) varied between $1,2,4,15$ and $30^{\circ}$, and could be aligned in the horizontal, diagonal (two directions: top-left to bottom-right, or bottom-left to topright) or vertical direction. Each of the 20 combinations of target amplitude and direction was presented for $20 \mathrm{~s}$, and the order of combinations was fixed. 
In the 'Light' paradigm, subjects were required to make spontaneous eye movements in the light. In the 'Dark' paradigm, subjects were required to make spontaneous eye movements in complete darkness. No stimuli were presented during these latter two paradigms.

\subsection{Procedure}

For each subject the paradigms were conducted in the following order: 'Target', 'Light', and 'Dark'. For each of the three paradigms: the subject was instructed about the task they were about to perform and was seated comfortably in front of the monitor. After successful calibration and check of the calibration (which was also recorded), the paradigm was started.

In the 'Target' paradigm, the subject was requested to change his point of gaze frequently between the two dots presented at a self-paced rate of about 1 saccade/s. Each pair of dots was presented for $20 \mathrm{~s}$, allowing for roughly 20 saccades to be made between targets, not including the small correction saccades. Before presentation of each pair of dots, a central dot was displayed in the center of the screen. This dot was used by the video system for automatic drift correction.

In the 'Light' paradigm, the subject was requested to make spontaneous shifts of gaze for approximately 5 min. Eye movement behavior was monitored and, if necessary, the subject was asked to make specific eye movements in order to obtain recordings throughout the tested oculomotor range.

The 'Dark' paradigm was highly similar to the 'Light' paradigm, except that the experimental room was darkened completely.

Total duration of the whole experiment was about 30 $\min$.

\subsection{Data analysis}

The data were analyzed offline. Left and right eye data were treated independently. Data points approximating the horizontal and vertical saturation levels of the coil system $\left( \pm 20^{\circ}\right)$ were discarded from the analysis.

Two-dimensional coil and video data were transformed into Euler angles with respect to the horizontal and vertical axes, respectively, taking the distance to the monitor into account, with position $(0,0)$ corresponding to straight ahead and the center of the screen, respectively. Note that these Euler angles do not represent true ocular rotation and introduce small errors when estimating, for example, real eye velocities. However, since this transformation to Euler angles was applied to both the coil and video signals this will not affect their mutual comparison.

The recordings were aligned in time manually, using a single large saccade recorded by both systems, by shifting that saccade in time to overlap graphically as good as possible. The mean difference in the recordings was subtracted from the video signal, hence, correcting roughly for the positional offset between 'straight ahead' (origin of the coil system) and the 'center of the screen' (origin of the video system). Horizontal and vertical eye orientation data of both the coil and video signal were both low-pass filtered using a fourth order Butterworth filter with a cut-off frequency of $120 \mathrm{~Hz}$. Instantaneous velocity signals were calculated as the difference in eye position between two consecutive sample points divided by the inter-sample interval ( $2 \mathrm{~ms}$ for the coil system and $4 \mathrm{~ms}$ for the video system).

We analyzed saccadic eye movements and the fixations in between gaze shifts. Slow gaze shifts such as possible slow phases of nystagmus in the 'Dark' condition' were not taken into further account.

\subsection{Fixation analysis}

Fixations were marked automatically in the coil signal as having a velocity of less than $0.2^{\circ} / \mathrm{s}$ for at least $250 \mathrm{~ms}$. The parts in the video signal, corresponding to these fixations, were marked. At these markers signal traces were extracted from the coil signal and video signal. Horizontal and vertical positions were calculated from the two signals independently, as the mean eye position during the fixation. Comparison of these fixation positions between the two systems was done to test the linearity of the video system and estimate the position drift over time.

\subsection{Saccade analysis}

Since saccades are the fastest eye movements, they provide a challenge for the dynamical properties for any eye tracking system. Therefore, we compared kinematical, as well as metric properties of saccadic eye movements measured by the two systems.

Saccades were marked automatically in the coil signal, using a velocity threshold of $50^{\circ} / \mathrm{s}$ and checked manually to ensure proper detection. The parts in the video signal, corresponding to the saccades marked in the coil signal, were marked. Around these markers signal traces were extracted from the coil signal and video signal. The following individual saccade analysis was applied independently to the coil and the video signals.

Saccadic onset and offset sample points for each saccade were determined using a (lower) velocity criterion of $30^{\circ} / \mathrm{s}$. The onset sample point was defined as the sample point closest to $4 \mathrm{~ms}$ before this level was crossed. The offset sample point was taken to be closest to $4 \mathrm{~ms}$ after eye velocity had dropped below this criterion. 
For every detected saccade, the following saccadic parameters were determined: horizontal and vertical amplitude, duration and peak velocity.

Horizontal and vertical amplitude was calculated as the difference in horizontal and vertical eye position at the time of saccadic onset and offset. Horizontal and vertical amplitudes were transformed to polar coordinates, yielding the direction from the starting point of the saccade and the radial amplitude of the saccade (i.e. the Euclidian distance between starting point and endpoint of the saccade).

Duration was calculated as the difference in time of the offset and onset of the saccade, and the maximum velocity reached during the saccade was taken as the peak velocity.

\subsection{Parameter comparison}

For each of the seven eyes recorded, and for each parameter, the values calculated from the video system were fitted as a linear function of the values calculated from the coil system, according to:

$P_{\mathrm{VIDEO}}=A P_{\mathrm{COIL}}+B$,

where $P_{\text {VIDEO }}$ are the individual parameter values as calculated from the video signal, $P_{\mathrm{COIL}}$ are the individual parameter values as calculated from the coil signal, $A$ is the slope of the fit and $B$ the intercept. Correlations $\left(R^{2}\right)$ were calculated.

Means and standard deviations of these slopes and intercepts that are presented in the remainder of this paper were calculated per parameter across the three paradigms and the seven eyes recorded.

\subsection{Saccade size effects}

In order to investigate effects of saccade size on the variability of the accuracy of the video system, the proportion difference $\left(\left(P_{\mathrm{COIL}}-P_{\mathrm{VIDEO}}\right) / P_{\mathrm{COIL}}\right)$ was calculated for each saccade. Saccades were binned and standard deviations within each bin were calculated for the three characteristics taken into account (radial amplitude, duration and peak velocity).

\subsection{Main sequence analysis}

Finally, we determined the relation between saccade amplitude and peak velocity, and the relation between saccade amplitude and duration, i.e. the so-called 'main sequence' relations (Bahill et al., 1975). Through the amplitude-peak velocity relations we fitted an exponential function:

$V_{\text {peak }}=S\left(1-\mathrm{e}^{-\mathrm{Amp} / \lambda}\right)$,

where ' $V_{\text {peak }}$ ' is the fitted peak velocity $\left({ }^{\circ} / \mathrm{s}\right)$, 'Amp' is the saccade amplitude $\left({ }^{\circ}\right)$, and ' $S$ ' and ' $\lambda$ ' are the fitted parameters (the saturation level $(\% / s)$ and the length constant $\left({ }^{\circ}\right)$, respectively). These fits used the NelderMead simplex method.

The amplitude-duration relationship was fitted by a straight line, as determined by linear regression, according to:

Dur $=$ Offset $_{\mathrm{AD}}+$ Gain $_{\mathrm{AD}}$ Amp,

where 'Amp' is the amplitude of the saccade $\left(^{\circ}\right)$ and 'Dur' is the duration of a saccade (ms). Offset ${ }_{\mathrm{AD}}$ is the intercept with the $y$-axis (ms), and Gain $_{\mathrm{AD}}$ is the slope of the line $\left(\mathrm{ms} /{ }^{\circ}\right)$.

\section{Results}

For all the results presented below, it is assumed that the coil signal is perfect, which might not be entirely true.

Fig. 1 shows $20 \mathrm{~s}$ of the horizontal and vertical eye position of the left eye of subject $B$ as recorded with the video and the coil system simultaneously.

\subsection{Comparison of fixation positions}

Fig. 2 compares the eye position output during fixations of both systems.

Throughout the measured range, there is a good linear relationship between the fixations that are simultaneously measured in the coil and video signal. This indicates that neither the video nor the coil tends to saturate at larger eccentricities. Please note that the coil recording range was limited to $40^{\circ}$ horizontally and vertically.

The well-correlated fits with slopes near one (horizontal: $1.071 \pm 0.062$; vertical: $1.029 \pm 0.048$ ) indicate that the video system is capable of recording fixation positions highly accurate over the used range of 40 by $40^{\circ}$ of visual angle. Individual offsets, albeit on average close to zero (horizontal: $0.630 \pm 0.790$; vertical: $-0.720 \pm 1.510)$ are likely to indicate small misalignments between the 'center of the screen', i.e. the origin for the video system, and 'straight ahead', i.e. the origin for the coil system.

To estimate the stability over time, horizontal and vertical fixation errors were defined as the difference in fixation positions of the coil and the video system (Fig. 3). A linear fit of these errors as a function of the recording time yields slopes near zero $(0.182 \pm 0.249)$, which indicates that there is no systematic change and high stability in the output of the video recording system over a time course of several minutes. Since we did an automatic drift correction between trials in the 'Target' paradigm, these data were discarded from this analysis. The average standard deviation for a recording of $5 \mathrm{~min}$, with eye positions throughout the whole 


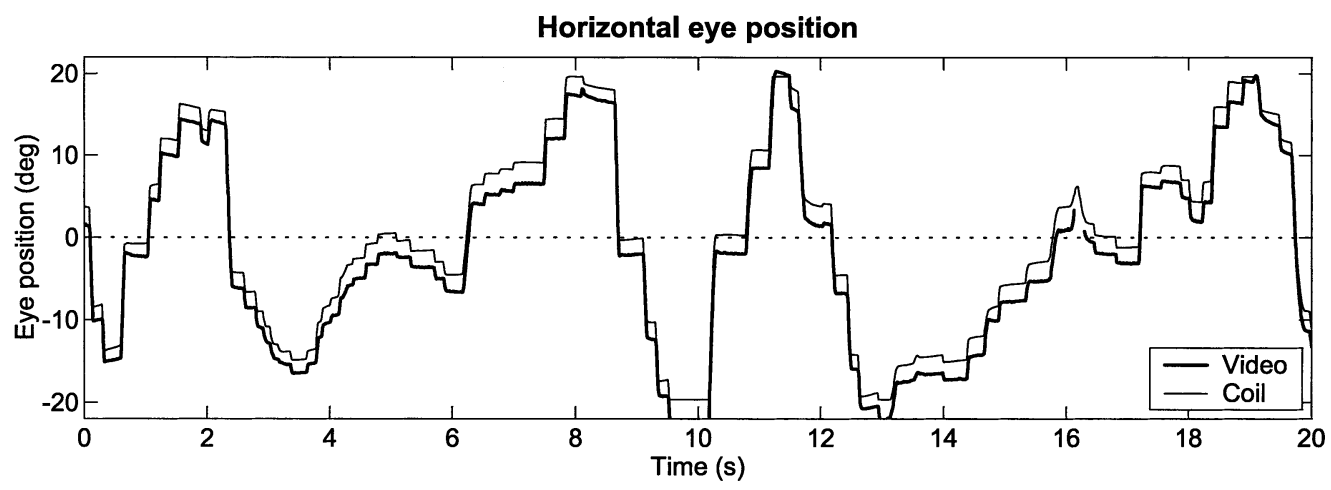

Vertical eye position

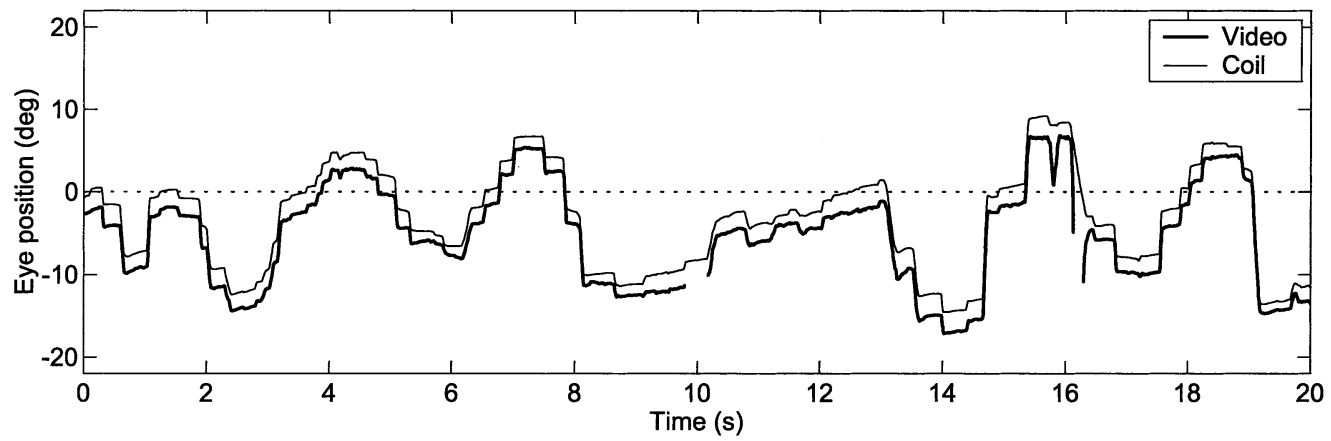

Fig. 1. Simultaneous recording by the video and coil system of the horizontal (top panel) and vertical (bottom panel) left eye position of one subject for $20 \mathrm{~s}$ in the light paradigm. Positive positions are to the right and upwards. Measurement range for the coil system was $\pm 20^{\circ}$ horizontally and vertically (see, e.g. saturation of the coil signal at 10,12,13 and $19 \mathrm{~s}$; the saturation at $10 \mathrm{~s}$ is a blink as indicated by the absence of vertical signal from the video signal). For clarity a small vertical offset has been added to the video signal.
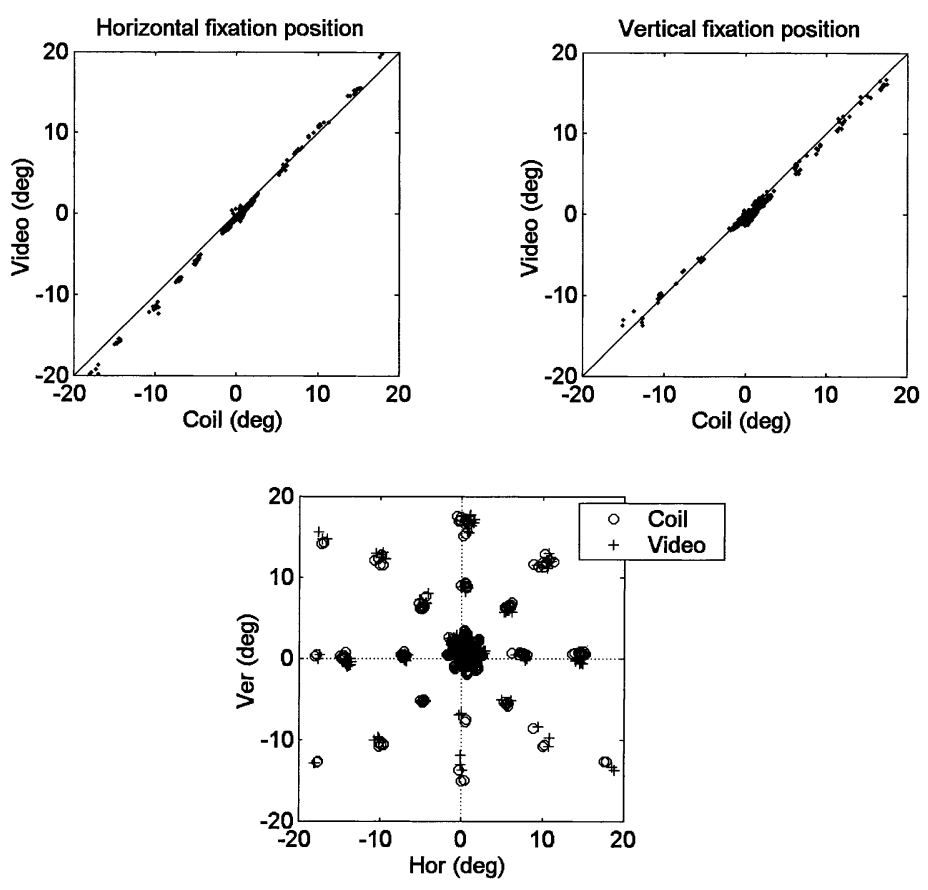

Fig. 2. Top panels: horizontal (left panel) and vertical (right panel) fixation positions of the video system as a function of the output of the coil system. Bottom panel: vertical versus horizontal fixation positions as estimated from the coil and video signal independently. Data of one subject in the 'Target' paradigm, including the check of the calibration. 


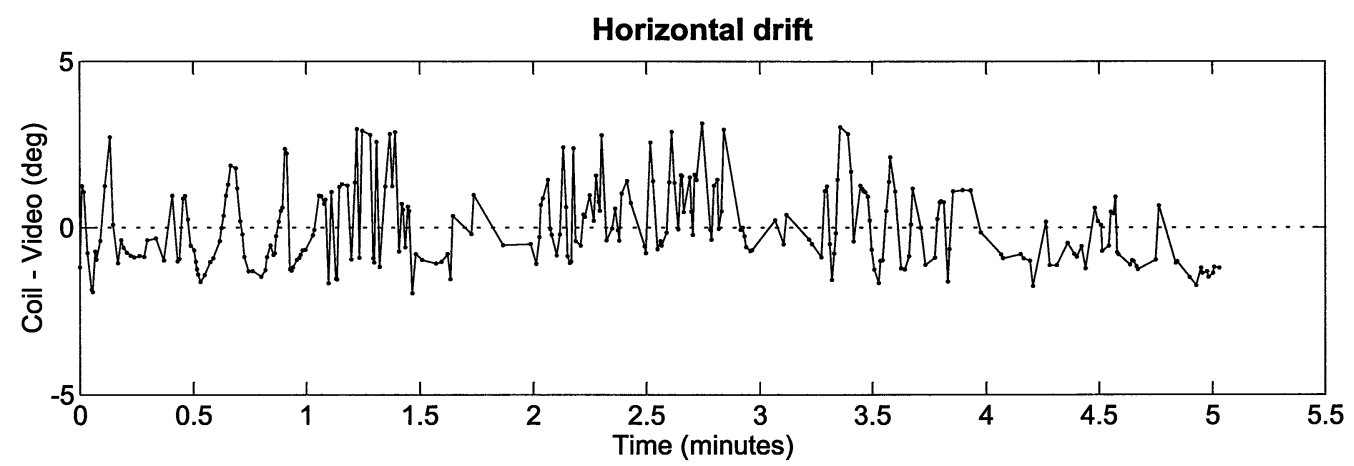

Vertical drift

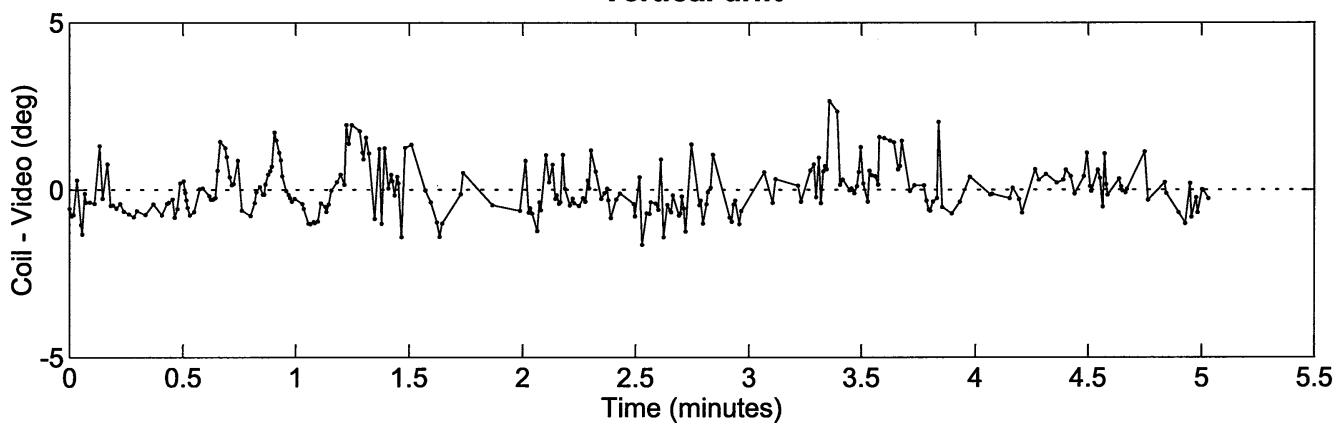

Fig. 3. The horizontal and vertical drift, defined as the difference between coil and video fixation position over time, for one subject in the light paradigm. Fixations were taken from the whole range of the coil system.
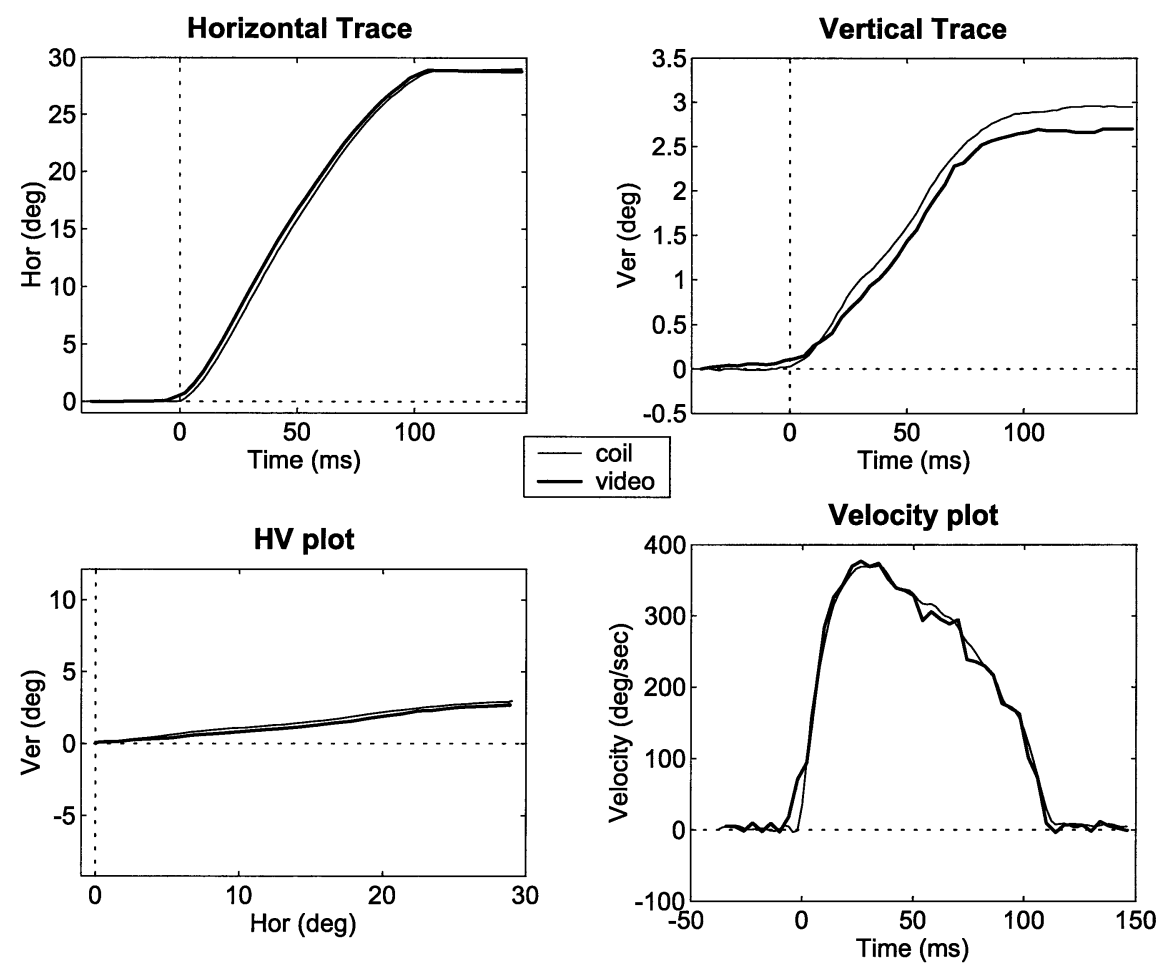

Fig. 4. A horizontal saccade of about $30^{\circ}$ to the right. The peak velocity profile is highly asymmetric. 
oculomotor range $\left(40\right.$ by $40^{\circ}$ ) was $0.98^{\circ}$ for the horizontal errors and $1.05^{\circ}$ for the vertical errors, across the seven eyes in two experiments.

\subsection{Comparison of saccade metrics and kinematics}

To illustrate the data set for the saccades, the video and coil eye position traces and eye velocity profiles of an individual saccade are plotted in Fig. 4.

With respect to saccade metrics, an example of the values for the measured video and the coil amplitudes can be seen in Fig. 5. The linear fit of all the subjects and paradigms yielded slopes near one for horizontal $(1.080 \pm 0.063)$ and vertical amplitudes $(1.041 \pm 0.054)$. The slopes were close to unity for the saccade direction
$(0.997 \pm 0.011)$ and the radial amplitudes $(1.067 \pm$ $0.055)$.

An example of correlation between the video and the coil for the saccadic peak velocity and duration is shown in Fig. 6. Saccadic peak velocity and saccadic duration are well correlated yielding slopes near unity $(1.075 \pm 0.072$, and $1.030 \pm 0.028$ for peak velocity and duration, respectively).

The effect of saccade size on the differences between the coil and video measurement is shown in Fig. 7. From these graphs one can see that, if one assumes that all differences are due to the video system, the relative accuracy of saccadic properties through the video system is less for small saccades having shorter durations and lower peak velocities, than for larger saccades.
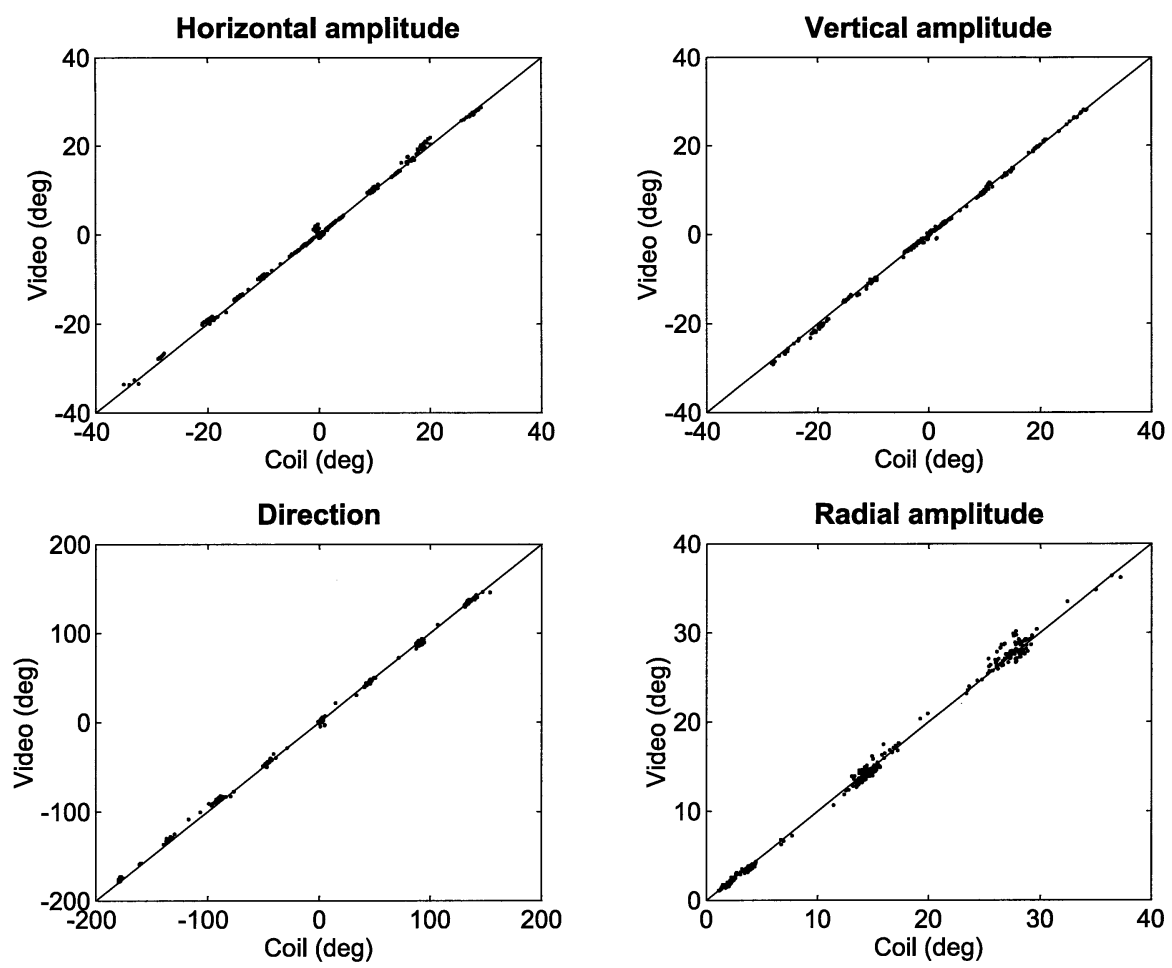

Fig. 5. Saccade metrics: values of video as a function of the values of coil.
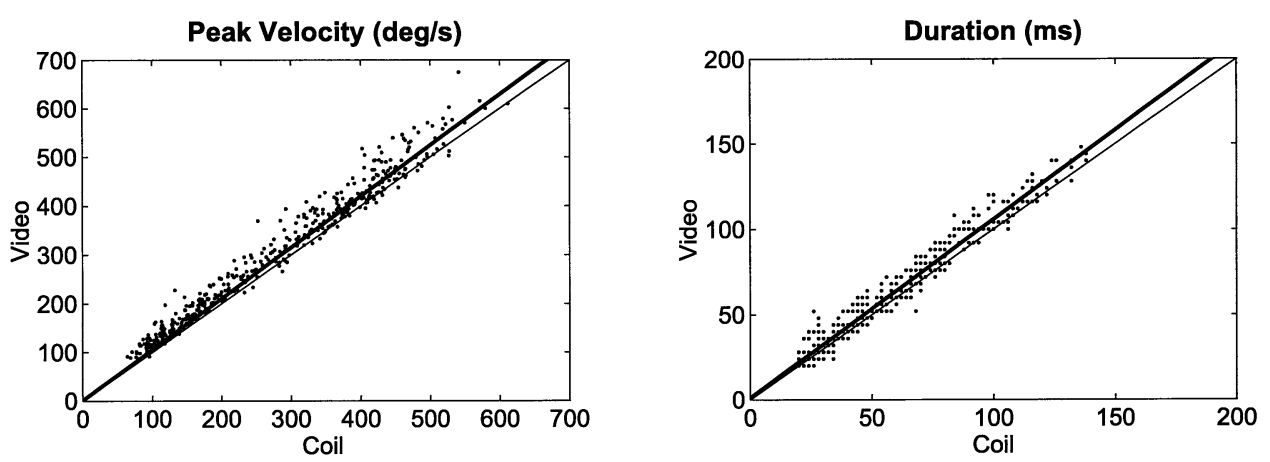

Fig. 6. Correlation of saccade dynamics between coil and video output: peak velocity, duration for one subject in the 'Dark' paradigm. The best linear fit (thick lines) deviates from the unity line (thin lines). 

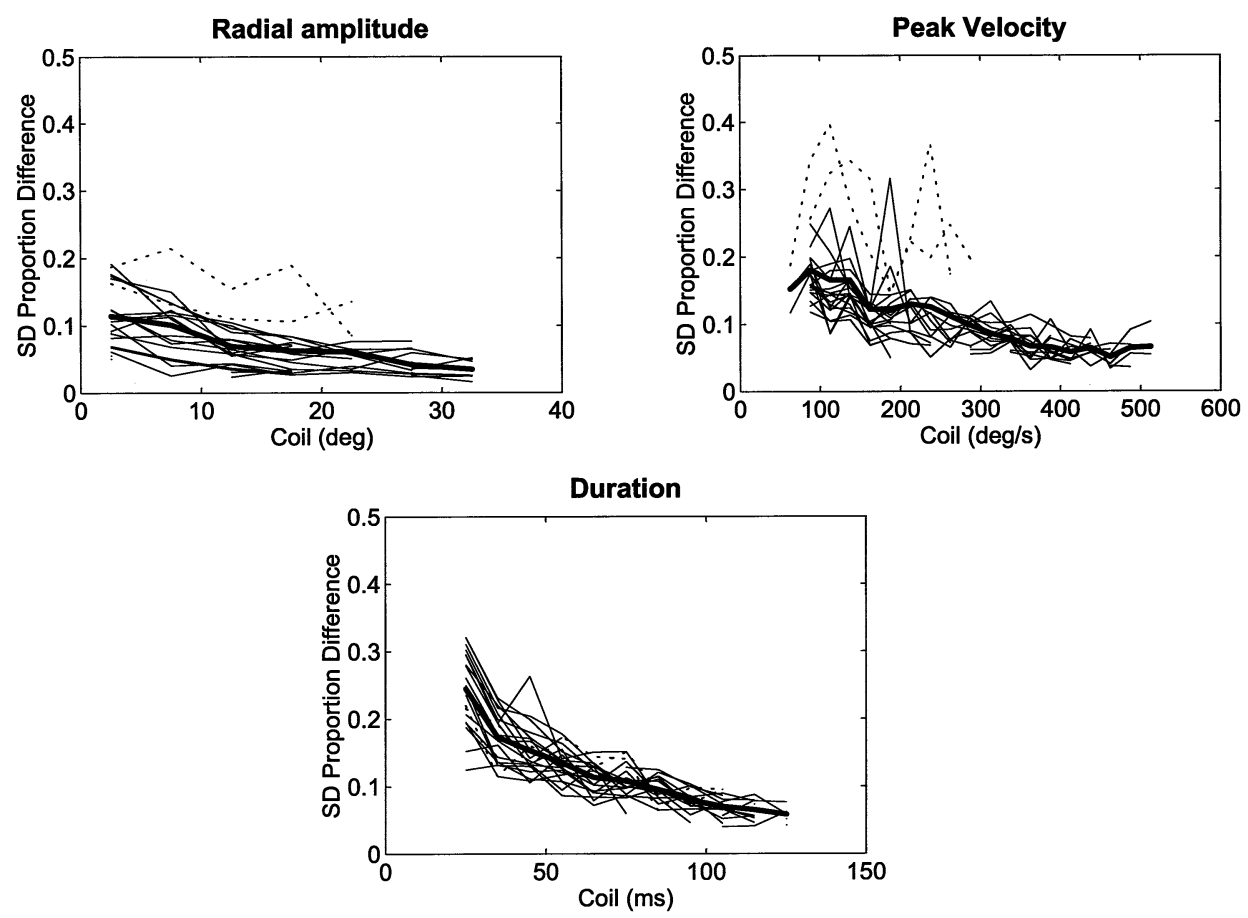

Fig. 7. The standard deviation of the proportion difference (i.e. $\left.\left(P_{\mathrm{COIL}}-P_{\mathrm{VIDEO}}\right) / P_{\mathrm{COIL}}\right)$ as a function of coil values for the three parameters of saccadic eye movements. Thick lines are the averages over the recordings from seven eyes (thin lines). The dotted lines are the left and right eye of one subject with spontaneous nystagmus in the dark, and therefore showing a large number of small saccades.

Table 1 summarizes the results and shows the means and standard deviations of the linear fit parameters Eq. (1) for each of the three paradigms, averaged over the seven eyes recorded. Slopes near one and intercepts near zero indicate that the parameters are measured equally well by the video system and the coil system.

Furthermore, the correlation between coil and video did not depend on the paradigm, in general. This means that the video system works equally well in the light and in the dark, despite differences in pupil diameter (which was, according to the pupil diameter output of the EyeLink system, on average twice at large in the dark than in the light).

\subsection{Comparison of main sequence}

The main sequence relationships between amplitude and peak velocity Eq. (2), amplitude and duration Eq. (3) were determined independently for both systems. An example of these two relationships for the coil and video outputs can be seen in Fig. 8 .

For each of the four parameters of the amplitudepeak velocity and amplitude-duration relationships, the values as estimated by the video system were plotted against the values as estimated by the coil system for each of the seven eyes recorded (Fig. 9).

Linear fits of video values as a function of coil values were calculated for each of these four parameters. For the amplitude-peak velocity relationship, linear fits for the two fit parameters yielded:

$$
\begin{aligned}
& S_{\mathrm{VIDEO}}=0.90 S_{\mathrm{COIL}}+68.52 \quad\left(R^{2}=0.97\right), \\
& \lambda_{\mathrm{VIDEO}}=0.80 \lambda_{\mathrm{COIL}}+0.86 \quad\left(R^{2}=0.85\right) .
\end{aligned}
$$

The linear fits for the two fit parameters of the amplitude-duration relationship, yielded:

$$
\begin{aligned}
& \text { Gain }_{\mathrm{AD}, \mathrm{VIDEO}}=0.82 \mathrm{Gain}_{\mathrm{AD}, \mathrm{COIL}}+0.60 \quad\left(R^{2}=0.99\right), \\
& \text { Offset }_{\mathrm{AD}, \mathrm{VIDEO}}=0.85 \mathrm{Offset}_{\mathrm{AD}, \mathrm{COIL}}+7.89 \quad\left(R^{2}=0.71\right) .
\end{aligned}
$$

\section{Discussion}

Simultaneous recordings of the two-dimensional movement of the eye by the scleral search coil and a video-based eye tracking system were compared for their measurement of metrics and kinematics of saccadic eye movements. Properties of fixations and saccadic eye movements as measured by the two systems independently were compared as well as the several relationships that exist between saccade amplitude, duration and peak velocity (Eqs. (2) and (3)).

Comparison of fixation positions showed that the video and coil signals were highly correlated in a linear way for the whole measurement range of $\pm 20^{\circ}$ horizontally and vertically. Furthermore, no systematic 
Table 1

The slopes and intercepts of the fits between the coil and the video system for the saccadic parameters in each of the three paradigms

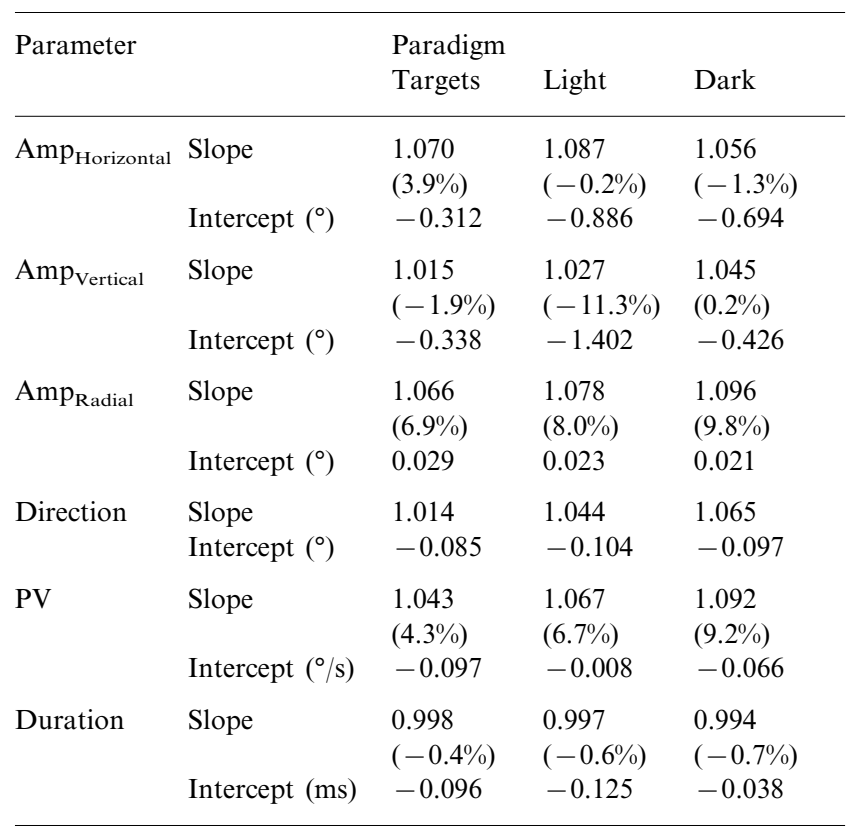

The number between brackets are the percentages by which the video signal differs from the coil signal for each of the relevant parameters, assuming a saccade with a size of $10^{\circ}$ having a peak velocity of $300^{\circ} / \mathrm{s}$ and a duration of $50 \mathrm{~ms}$.

drift of the output was observed in the video system with respect to the coil output, indicating that this system has a high stability in tracking the position of the eye. However, if one assumes that the coil method does not contribute to fixation error between the coil and video system (i.e. all the differences are due to the video system) the observed standard deviations in fixation error suggests that this video system should be treated with care when the accuracy of fixation position is required to be smaller than $1^{\circ}$.
The comparison of saccade parameters showed that the measurements of saccadic amplitude, duration, peak velocities were highly correlated between the coil and video system. These high correlations indicate that the video system was capable of tracking even the fastest eye movements with great accuracy (see, e.g. Fig. 4).

Finally, comparison of the main sequence, i.e. the amplitude-duration and amplitude-peak velocity relationships (Bahill et al., 1975) showed that these saccadic characteristics were similar between the coil and video recordings. The peak velocity saturation level was slightly overestimated by the video system. So, the video system should be used with care if one wants to estimate the true velocities of the eye, but is a good solution if one wants to compare differences in main sequences under different conditions. The coil and the video system correlate highly linear for all of the metric and kinematical parameters. Depending on the research question, this suggests that the video system can be reliably used in measuring differences in (most) saccadic properties between, e.g. conditions and/or groups.

However, the results do show discrepancies between the video system and the coil system. In the present paper, these were treated as errors of the video system. One might argue that this is not necessarily true and that the coil system itself might introduce errors, although the Remmel system is regarded as highly accurate (Collewijn, 1998). For instance, the observation that saccadic peak velocity was slightly higher in the video system than in the coil system might be related to the visco-elastic coupling between the annulus and the cornea. This would suggest that the coil motion is a filtered version of the actual eye movement, thereby underestimating true peak velocity of saccades.

Another explanation might be a high frequency noise in the video signal (e.g. due to the lower spatial and temporal resolutions of the video system), which could
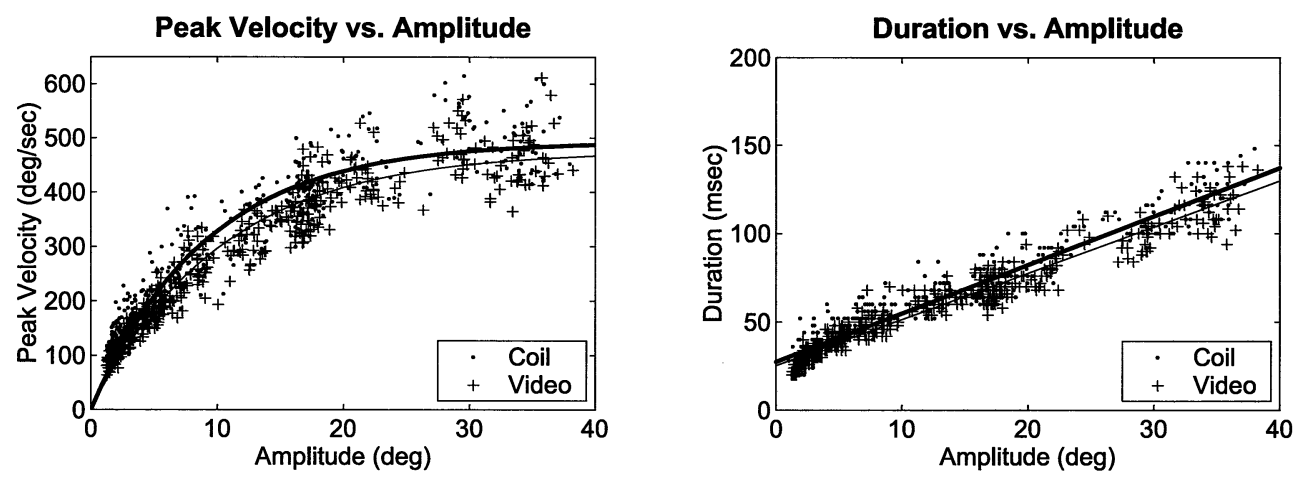

Fig. 8. An example of the two relationships for the coil and video output for the left eye of one subject in the 'light' paradigm between the amplitude, peak velocity, and duration. Each point represents one saccade. Thick lines indicate the fits between parameters from the video signal; thin lines those from the coil signal. 

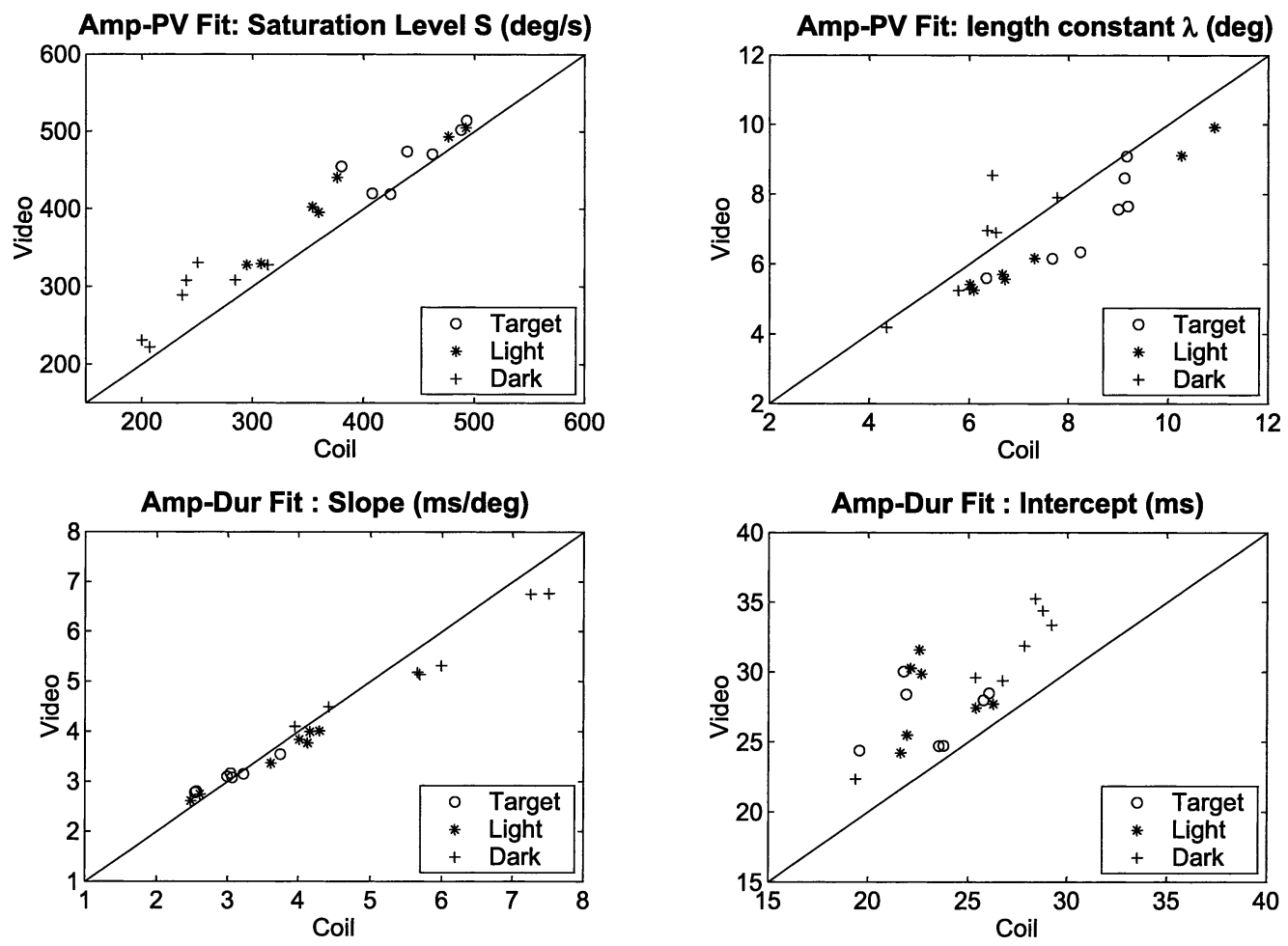

Fig. 9. Video versus coil fit parameters of the main sequence relationships (Eqs. (2) and (3)), for each of the three paradigms. For each paradigm the parameters for the seven eyes recorded are plotted.

increase the peak velocity by the half-width of this noise, when estimated using a simple maximum function. Therefore, using a simple maximum function could be misleading. However, estimating peak velocity by fitting a gamma function (Van Opstal and Van Gisbergen, 1987), or a fitting 2nd order polynomials through the velocity signal of a saccade instead of using two-point differences in the raw data, yielded no different results than those presented here. Therefore, it seems unlikely that such a high frequency noise contributed significantly to the higher peak velocities in the video signal.

Three aspects of the video-oculographic system were not studied here. Firstly, a possible delay between an eye movement and an auxiliary input, signaling, e.g. a stimulus change was not investigated. Such information might important if one wants to study latencies and phase shifts. Secondly, the present system does not track a corneal reflection, and cannot compensate for any relative movement between cameras and the eyes. The implications of tracking only the center of the pupil were not tested. The tested video system might have a better signal-to-noise ratio than other video-oculographic devices in which the noise is the sum of the noise from the separate pupil and corneal reflection signals. Thirdly, the head tracker system, that allows for eye tracking in a head free situation was not tested. These three aspects deserve further investigations in the future.

In conclusion, this video system seems to be an alternative for the scleral search coil technique, especially in cases where the scleral search coil technique is not applied easily, for instance, when children or patients are to be tested on their oculomotor behavior. However, the coil system still has, of course, several advantages, e.g. in recording torsional eye movements $(3 \mathrm{D}$ eye movements) with higher sample rates than $50 \mathrm{~Hz}$ (the current limit of torsional video-oculography), and in recording $2 \mathrm{D}$ eye movements with very high sample rates, e.g. in order to estimate differences in saccadic onset profiles between the two eyes (Collewijn, 2001). Furthermore, the low sample rate may limit the correct calculation of saccade characteristics, especially peak velocities (Juhola and Pyykko, 1987) and skewness (Van Opstal and Van Gisbergen, 1987). This is mainly a problem for small saccades as can be seen in Fig. 7.

In conclusion, the main disadvantage of the video method is its low sample rate. The relatively noisier estimate of all parameters of small saccades can be attributed to this low sampling frequency. Therefore, a video system that is to replace the coil system is preferred to sample with higher sampling rates.

Furthermore, it is important to emphasize that characteristics of the video system used in the present study (EyeLink version 2.04) may or may not apply to video systems marketed by other companies. Secondly, detailed information about hardware and software (e.g. internal filtering) might be unavailable, since most of the video- 
oculographic systems on the market uses proprietary techniques. Moreover, the hardware and software of such eye tracking systems might change at any moment, which is likely to yield different characteristics that remain unknown unless properly tested and compared to the accepted gold standard in oculomotor research.

\section{Acknowledgements}

For this work J.N. van der Geest was supported by NWO-MW-903-68-394 and EMCR-RF-194.664-2000181. The authors thank Han Collewijn for the use of his experimental setup.

\section{References}

Bahill AT, Clark MR, Stark L. The main sequence, a tool for studying human eye movements. Math Biosci 1975;24:191-204.

Collewijn H. Interocular timing differences in the horizontal components of human saccades. Vision Res 2001;41:3413-23.

Collewijn H. Eye movement recording. In: Carpenter RHS, Robson JG, editors. Vision Research: a Practical Guide to Laboratory Methods. Oxford University Press, 1998:245-85.
Collewijn H, van der Mark F, Jansen TC. Precise recording of human eye movements. Vision Res 1975;15:447-50.

Deubel H, Bridgeman B. Fourth Purkinje image signals reveal eyelens deviations and retinal image distortions during saccades. Vision Res 1995;35:529-38.

DiScenna AO, Das V, Zivotofsky AZ, Seidman SH, Leigh RJ. Evaluation of a video tracking device for measurement of horizontal and vertical eye rotations during locomotion. J Neurosci Methods 1995;58:89-94.

Juhola M, Pyykko I. Effect of sampling frequencies on the velocity of slow and fast phases of nystagmus. Int $\mathbf{J}$ Biomed Comput 1987;20:253-63.

Remmel RS. An inexpensive eye movement monitor using the scleral search coil technique. IEEE Trans Biomed Eng 1984;31:388-90.

Robinson DA. A method of measuring eye movement using a scleral search coil in a magnetic field. IEEE Trans Biomed Electron 1963;10:137-45.

Sheena D, Borah B. Compensation for some second-order effects to improve eye position measurements. In: Fisher BF, Monty RA, Sender JW, editors. Eye Movements: Cognition and Visual Perception. Hillsdale New Jersey, 1981.

Stahl JS, van Alphen AM, De Zeeuw CI. A comparison of video and magnetic search coil recordings of mouse eye movements. J Neurosci Methods 2000;99:101-10.

Stampe DM. Heuristic filtering and reliable calibration methods for video-based pupil-tracking systems. Behav Res Methods Instrum Comput 1993;25:137-42.

Van Opstal AJ, Van Gisbergen JA. Skewness of saccadic velocity profiles: a unifying parameter for normal and slow saccades. Vision Res 1987;27:731-45. 